\title{
Phytochemical analysis of ionic liquid-Graviola (Annona muricata) fruit extract and its acute toxicity on zebrafish early-life stages
}

\author{
Djabir Daddiouaissa ${ }^{\mathrm{a}}$, Azura Amid ${ }^{\mathrm{b} *}$, Syahida Ahmad ${ }^{\mathrm{c}}$, Ahmed A. M. Elnour ${ }^{\mathrm{a}}$ \\ ${ }^{a}$ Biotechnology Engineering Department, Kulliyyah of Engineering, International Islamic University Malaysia (IIUM), P. O. Box 10, \\ Gombak. 50728 Kuala Lumpur, Malaysia \\ ${ }^{b}$ International Institute for Halal Research and Training (INHART), Level 3, KICT Building, International Islamic University Malaysia \\ (IIUM), Jalan Gombak, 53100 Kuala Lumpur, Malaysia \\ 'Department of Biochemistry, Faculty of Biotechnology \& Biomolecular Sciences, Universiti Putra Malaysia, 43400 UPM Serdang, \\ Selangor, Malaysia
}

Received 2nd January 2020 / Accepted 6th June 2020

\begin{abstract}
Annona muricata, commonly known as soursop and Graviola, is a member of the Annonaceae family. Some of its phytochemicals were reported to have a neurotoxicity effect causing neurodegenerative diseases. However, different parts of this tree have been used for ages in traditional medicine due to their biological activities, such as anti-inflammatory, antimicrobial, antioxidant and anticancer effects. This study aimed to qualitatively screen the crude ionic liquid-Graviola fruit extract (IL-GFE) phytochemical composition, assess its acute toxicity and determine the lethal concentrations using zebrafish (Danio rerio) embryos. IL-GFE contains acetogenins, alkaloids, phenols, terpenoids, tannins and flavonoids. Acute toxicity effects of IL-GFE on zebrafish embryos were observed from 24 to 120 hours of post fertilisation (hpf). The survival rate, $\mathrm{LC}_{50}$, sublethal endpoints and effect of IL-GFE on the heart rate of zebrafish larvae were assessed. Results showed that the lethal concentration $\left(\mathrm{LC}_{50}\right)$ of the crude IL-GFE was 173.45 $\mu \mathrm{g} / \mathrm{mL}$. Interestingly, no significant changes on the morphology of the treated zebrafish were observed at a concentration of $125 \mu \mathrm{g} / \mathrm{mL}$. However, the heart rate of zebrafish larvae at $96 \mathrm{hpf}$ was significantly decreased by $33.76 \%$ after treated with crude IL-GFE at $125 \mu \mathrm{g} / \mathrm{mL}(119.00 \pm 4.72$ beats/min) as compared to the untreated group $(179.67 \pm 4.66$ beats $/ \mathrm{min})$. This preliminary finding showed that crude ionic liquid-Graviola fruit extract and its phytoconstituents might have the potential to be developed as a food supplement or herbal product. However, further tests need to be conducted to evaluate its medicinal properties and adverse effects on organisms of higher orders.
\end{abstract}

Keywords: acute toxicity, Annona muricata (Graviola), phytochemicals, teratogenicity, zebrafish assay

\section{INTRODUCTION}

Medicinal plants and their constituents have been used for a considerable period by many communities and civilisations to help humankind to maintain good health and treat different diseases (Yunus et al., 2010). Extensive studies are applied to evaluate the application and formulation of medicinal plants and their bioactive compounds in folk medicine with significant concerns about their efficiency and safety. Despite all their essential pharmacological functions, some of the phytoconstituents could be causing potential toxicity, teratogenicity

*Author for correspondence: Azura Amid, International Institute for Halal Research and Training (INHART), Level 3, KICT Building, International Islamic University Malaysia, Jalan Gombak, 53100 Kuala Lumpur, Malaysia. Email-azuraamid@iium.edu.my 
(teratogen agent can cause or induce morphological abnormalities), carcinogenicity, and mutagenicity as an individual compound or in combinations (Ng'uni et al., 2018; Romagosa et al., 2016). Thus, studying the toxic effect and teratogenicity of medicinal plants through their potential extracts is pivotal. The conventional animal models used for acute toxicity studies are mammals, including rats, mice and rabbits as well as flies and worms which are used for specific genetic and molecular studies (Lieschke and Currie, 2007).

Zebrafish (Danio rerio) has been used as an alternative model in biomedical and toxicology research due to the tremendous advantageous features among other vertebrates' model. Zebrafish is a small tropical freshwater fish, belonging to the minnow (Cyprinidae) family under the order of Cypriniformes (Parichy et al., 2009). This fish is characterised by its transparency at the early stage of development, high embryos production number, quick maturation and high similarity with humans genome (over 80\% genomic homology) as reported earlier by Oliver et al. (2011). Hence, higher genomic homology enables a significant correlation between the obtained data from the two species (human and zebrafish). Moreover, Ong et al. (2009) reported that zebrafish is widely used in disease studies and drug discovery due to the lower cost of handling compared to other models such as rodents; and they grow at the minimal environmental conditions and small space (e.g. aquarium). For these reasons, reducing animal trails is being necessary.

Reducing animal testing is one of the main goals of the Food and Drug Administration (FDA), which is stated in ISO 10993, hence zebrafish is therefore considered to be an optimum choice to replace rodent model and other animals testing. Since zebrafish can produce a thousand of embryos at one time compared to a rodent which produces at best only a dozen, consequently it is less time consuming and low amount of drug testing needed (Hill et al., 2005). Recently, zebrafish have been used to identify the toxicity of nanoparticles (Abdul Ghafor et al., 2020), antibiotics (Oliveira et al., 2020) and metal (Bai and Tang, 2020). The acute toxicity of a substance on zebrafish can be conducted by a morphological observation to assess the lethal endpoints such as malformations (death/coagulation, missing heartbeat, no tail detachment, missing somites, no spontaneous movement) and non-lethal malformations (spine deformation, yolk sac oedema, no pigmentation, low or missing of blood circulation and incomplete eye development) (Kapp et al., 2006).

Annona muricata (Graviola) fruit is a member of the Annonaceae family that obtained much interest in the last decades due to its pharmaceutical potential. Previously, many studies reported that Annona muricata had been used in traditional medicine to treat many diseases such as diabetes, diarrhoea, fever, inflammation and stomach pain. Since then, the bioactivity and toxicity of this plant attracted the attention of many researchers as summarised by Coria-Téllez et al. (2018) and Daddiouaissa \& Amid. (2018). Numerous techniques are used to extract bioactive compounds from Graviola fruit such as Soxhlet and maceration extraction (Ragasa et al., 2012; Sun et al., 2017). These methods have shown low efficiency, environmental pollution and a long time that is required for extraction (Dai et al., 2011). On the other hand, a modern and more efficient technique of extraction such as microwave-assisted extraction (MAE) coupled with ionic liquids (ILs), has accelerated research in analytical chemistry as green and designer solvents. The structure of ionic liquids has a great influence on their physicochemical properties, which may have an impact on the extraction efficiency of targeted analytes (Tang et al., 2012).

Several studies assessed the acute toxicity, mutagenicity and neurotoxicity of the Graviola leave, and its phytoconstituents on Wistar rats and Swiss albino mice (Escobar-Khondiker et al., 2007; Guadaño et al., 2000; Lannuzel et al., 2003; Sousa et al., 2010). Nevertheless, no research was conducted regarding the toxicity level and teratogenicity effects of the crude ionic liquid Graviola fruit extract (IL-GFE) on developing zebrafish embryos. Therefore, this study aimed to identify the phytoconstituents of the crude ILGFE and to assess the toxicity level, determine the lethal concentration and measure the teratogenicity effects of this extract by using the apparent clinical signs and symptoms of developing zebrafish embryos. 


\section{MATERIALS AND METHODS}

\section{Chemicals and reagents}

In this study, chemicals and reagents that were used are laboratory grade. They were utilised to investigate the phytochemical composition and acute toxicity effect of IL-GFE on developing zebrafish embryos. The ionic liquid, one-butyl-3methylimidazolium chloride [C4MIM] $\mathrm{Cl}^{-}(96 \%)$ reagent was purchased from ALFA, (USA). DMSO and Keddie's reagent (3,5-Dinitrobenzoic acid) were obtained from Sigma-Aldrich (USA). Dragendorff reagent was purchased from Merk (Germany), sulphuric acid and ferric chloride were obtained from HmbG Chemical (Germany).

\section{Plant collection and preparation}

Samples of ripe Graviola fruit (DB3) were randomly collected during the season of 2017/2018 from the Adikafirdaus Resources Farm $\left(4.5921^{\circ} \mathrm{N}, 101.0901^{\circ} \mathrm{E}\right)$, Perak state, Malaysia. The fruit sample was identified and deposited in the herbarium with voucher number KAED/HBL/S1A047/2018/706 at the Kulliyyah of Architecture and Environmental Design (KAED), IIUM. The website (www.theplantlist.org) was used to check the name of the plant. Then, Graviola fruit samples were cleaned and the pericarp and seeds were removed. After that, the fruit pulp was lyophilised using a freeze drier (Christ Alpha, Germany) for $72 \mathrm{hrs}$ to ensure initial sample texture is preserved. The dried sample was then ground to a powder form using a commercial blender (Kinematics, Swiss) and stored until further analysis (Gyamfi et al., 2011).

\section{Graviola fruit extraction}

The Graviola fruit samples were extracted as described in our previous article (Daddionaissa et al., 2019). Briefly, one gram of the dried powder of the fruit pulp was mixed with $20 \mathrm{~mL}$ of 1-butyl3-methylimidazolium chloride at $0.5 \mathrm{~mol} / \mathrm{L}$. Then, the suspensions were heated in the microwave oven under irradiation power of $700 \mathrm{~W}$ for $3 \mathrm{~min}$. Next, the obtained extract (IL-GFE) was cooled down to $25^{\circ} \mathrm{C}$ and then filtered using Whatman No. $3 \mathrm{~mm}$ filter paper and transferred into a test tube. Then, lyophilised the sample using a freeze drier for 72 hrs (Bhan et al., 2017; Zhang et al., 2014).

\section{Phytochemical screening}

According to the procedure of Harborne (1998) with slight modifications, the crude ionic liquid Graviola fruit pulp extract was subjected to qualitative determination of phytochemical constituents.

\section{Detection of acetogenins}

Keddie's reagent test was performed to detect the presence of acetogenins by using the method reported by Vyas. (2010). One drop of 90\% ethanol in distilled water and two drops of $2 \%$ Keddie's reagent (3,5-dinitrobenzoic acid) were added to $2 \mathrm{mg}$ of crude IL-GFE. Then, a 20\% sodium hydroxide solution was added to alkalize the mixture. Formation of purple colour indicated the presence of $\beta$-unsaturated- $\gamma$-lactones that are commonly present in the annonaceous acetogenins.

\section{Detection of alkaloids}

Dragendorff reagent test was performed to detect the presence of alkaloids by dissolving $2 \mathrm{mg}$ of the sample (IL-GFE) in diluted hydrochloric acid, then $1 \mathrm{~mL}$ of Dragendorff reagent was added to the mixture. Formation of reddish/brown precipitate indicates the presence of alkaloids (Harborne, 1998).

\section{Detection of phenols}

Ferric chloride test was applied to detect the presence of phenols by treating $2 \mathrm{mg}$ of the sample (IL-GFE) with $1 \mathrm{~mL}$ of ferric chloride solution. Formation of bluish-black colour indicates the presence of phenols (Bhat et al., 2009; Harborne, 1984).

\section{Detection of flavonoids}

Alkaline reagent test was employed to detect the presence of flavonoids as described by Evans. (1996). A few drops of $1 \mathrm{M}$ sodium hydroxide $(\mathrm{NaOH})$ solution were added to $2 \mathrm{mg}$ of the extract (IL-GFE) in a centrifuge tube. A formation of an intense yellow colour which becomes colourless after adding a few drops of $\mathrm{HCl}$ indicated the presence of flavonoids. 


\section{Detection of saponins}

An emulsion formation test was performed to detect the presence of saponins as described by Ejikeme et al. (2014) and Zakaria et al. (2019). About $200 \mu \mathrm{L}$ of distilled water was added to 2 $\mathrm{mg}$ of sample (IL-GFE). Then, approximately 1 $\mathrm{mL}$ of olive oil was added to the mixture and shaken vigorously. Formation of emulsion indicated the presence of saponins.

\section{Detection of terpenoids}

Salkowski test was applied to detect the presence of terpenoids as described by Ayoola et al. (2008). About $0.5 \mathrm{~g}$ of the sample (IL-GFE) was mixed with $2 \mathrm{~mL}$ of chloroform. Concentrated sulphuric acid $(3 \mathrm{~mL})$ was added to form a layer. Formation of a reddish-brown colour of the interface indicates the presence of terpenoids.

\section{Detection of tannins}

The diluted ferric chloride test was applied to detect the presence of tannins as described by Zohra et al. (2012) with slight modifications. About $2 \mathrm{~mL}$ of deionised water was added to the same volume of IL-GFE sample extract then, 1 to 2 drops of ferric chloride solution were added. The formation of a dark green or blue-green colour indicates the presence of tannins.

\section{Acute toxicity of IL-GFE on zebrafish embryos/larvae}

The acute toxicity of the crude IL-GFE was analysed using zebrafish embryos/larvae (24 to $120 \mathrm{hpf}$ ) screening assay in a 96-well plate to observe their morphological changes at in vivo level, determine the $50 \%$ lethality concentration $\left(\mathrm{LC}_{50}\right)$ and compare it to the standard $\mathrm{LC}_{50}$ of the plant extract on zebrafish embryos/larvae.

\section{Zebrafish maintenance and embryo collection}

After spawning, zebrafish embryos were collected from the adults' zebrafish of the wild type ( $\mathrm{AB}$ strain). The zebrafish adults were raised and maintained by Danio Assay Laboratories Sdn. Bhd., Malaysia, under standard laboratory conditions of animal welfare in a flow-through tank with a light/dark photoperiod of $14 / 10 \mathrm{hrs}$ at $28^{\circ} \mathrm{C}$. The zebrafish embryos were handled in conformity with the related laws and institutional guidelines of the Institutional Animal Care and
Use Committee (IACUC), with an ethical approval number (UPM/IACUC/AUPR059/2018), Universiti Putra Malaysia. The embryos were collected from a spawning tank after 1-hour post fertilisation (hpf), rinsed with egg water, staged and maintained in a Petri dishes at $28 \pm 2{ }^{\circ} \mathrm{C}$ until $24 \mathrm{hpf}$ in which the time of exposure was started.

\section{Zebrafish embryos/larvae toxicity}

The embryos/larvae acute exposure assay was performed by following the guideline of OECD (2013) and Yan et al. (2016) with some modifications. After embryo quality control test was carried out, the live zebrafish embryos were randomly transferred into 96-well plates with one healthy embryo (24 hpf) per well and twelve embryos in each treatment group (3 replicates). Then, $200 \mu \mathrm{L}$ of Danio-Sprint M Embryo Media was added with either a crude IL-GFE sample or $0.1 \%$ DMSO as a control. The stock solution of the crude IL-GFE at a concentration of 50 $\mathrm{mg} / \mathrm{mL}$ was dissolved in $10 \% \mathrm{v} / \mathrm{v}$ DMSO. Then, the IL-GFE stock solution was serially diluted to 2 -fold at seven concentrations ranging from 7.81 $\mu \mathrm{g} / \mathrm{mL}$ to $500 \mu \mathrm{g} / \mathrm{mL}$. DMSO was maintained at $0.1 \% \mathrm{v} / \mathrm{v}$ in all sample concentrations. The vehicle of $0.1 \% \mathrm{v} / \mathrm{v}$ DMSO was considered as untreated (control) and was observed to be safe to the embryos. The exposure was continued in a temperature-controlled incubator at $28 \pm 1{ }^{\circ} \mathrm{C}$ at different stages of development from pharyngula period (24-48 hpf), hatching period (48-72 hpf) to early larval period (72-120 hpf) (Kimmel et al., 1995). Embryos/larvae were observed every 24 hrs to assess the toxicological endpoints such as dead/coagulation, hatching rate, tail detachment, somite formation and body curvature using an inverted microscope (Olympus MVX10, Germany). The angle of curvature in the effected larvae was determined using digital images and compared to the linear spinal axis of the untreated group. The curvature of the spinal cord was measured and graded as low $\left(0-45^{\circ}\right)$, medium $\left(46-90^{\circ}\right)$, maximum $\left(91-135^{\circ}\right)$ or severe (136$\left.180^{\circ}\right)$ as described by Pamanji et al. (2015). The heart rate of the larvae was assessed at $96 \mathrm{hpf}$ and recorded using an inverted microscope (Olympus MVX10, Germany) with a digital camera attached to the ocular lens and heartbeat was counted for 1 min using a stopwatch. The mortality and 
hatching rates were determined using equations 1 and 2.

$$
\begin{aligned}
& \text { Mortality rate }(\%)=\frac{\text { No. of dead embryosy }}{\text { Total embryos number }} \times 100(\text { Eq 1) } \\
& \text { Hatching rate }(\%)=\frac{\text { No. of hatched embryos }}{\text { Total embryos number }} \times 100(\text { Eq 2) }
\end{aligned}
$$

\section{Statistical analyses}

The acute toxicity experiments were conducted in triplicates, and the data were represented as the overall mean of three independent experiments \pm standard deviation (SD). The findings were analysed by GraphPad Prism (Version 7.00, GraphPad Inc., CA, USA) to carry out the statistical analysis. One-way ANOVA analysis of variance technique followed by Tukey's test was performed using Minitab software (Version 17, Minitab Inc., State College, PA, USA) to evaluate the significant differences between samples with a certain interval of $95 \%$ and $\mathrm{P}<0.05$ was considered significant.

\section{RESULTS}

\section{Phytochemical screening}

The qualitative phytochemical analysis conducted on the crude IL-GFE revealed the presence of some biologically active compounds, including acetogenins, alkaloids, flavonoids, tannins, terpenoids, and phenols that exhibit many therapeutic activities (Table 1). They were detected based on the specific colour formation, froth formation, precipitation and the ring formation between the two layers (Ojezele et al., 2016).

Table 1. Qualitative phytochemical components of the crude Graviola fruit extract.

\begin{tabular}{ccc}
\hline Phytochemical constituents & Test name & Crude IL-GFE \\
\hline Acetogenins & Keddie's reagent test & ++ \\
Alkaloids & Dragendorff reagent test & ++ \\
Phenols & Ferric chloride test & + \\
Flavonoids & Alkaline reagent test & ++ \\
Saponins & Emulsion formation test & - \\
Terpenoids & Sulphuric acid test & + \\
Tannins & Diluted ferric chloride test & + \\
\hline
\end{tabular}

Note: Negative $(-)$ and positive (+) signs showed the absence and presence of metabolites in the crude IL-GFE, with (++) showed strong color and $(+)$ mild color.

\section{In vivo zebrafish embryo/larvae toxicity assay}

The acute toxicity of the crude IL-GFE on developing zebrafish embryos was conducted by assessing the standard toxicological endpoints such as dead/coagulation, heartbeat, tail detachment, hatching rate, somite formation and body curvature from 24 to $120 \mathrm{hpf}$.

\section{Effects of crude IL-GFE on the survival rate of zebrafish embryos}

Embryos exposed to serial concentrations of ILGFE were assessed daily and compared with the control group at 24, 48, 72, 96 and $120 \mathrm{hpf}$. The survival rates $(\%)$ of the embryos in egg water and the treated embryos with different concentrations of IL-GFE were shown in Figure 1. Concentrations above $250 \mu \mathrm{g} / \mathrm{mL}$ of IL-GFE resulted in $100 \%$ mortality at $96 \mathrm{hpf}$. The concentrations of less than $62.5 \mu \mathrm{g} / \mathrm{mL}$ did not show any significant mortality on developing zebrafish embryos until the end of exposure time at $120 \mathrm{hpf}$.

The $\mathrm{LC}_{50}$ value for IL-GFE was indicated using the statistical estimation of the extract $(\mu \mathrm{g} / \mathrm{mL})$ required to induce $50 \%$ of the dead population of developing zebrafish embryos using linear regression equation. Figure 2 represents the estimation of the $\mathrm{LC}_{50}$ value using the percentage of survival rate $(\%)$ of the embryos vs concentrations of crude IL-GFE $(\mu \mathrm{g} / \mathrm{mL})$ as described by Scarlett et al. (2013) and Yan et al. (2016). Based on the regression equation, the $\mathrm{LC}_{50}$ of the crude IL-GFE was calculated to be $173.45 \mu \mathrm{g} / \mathrm{mL}$. 


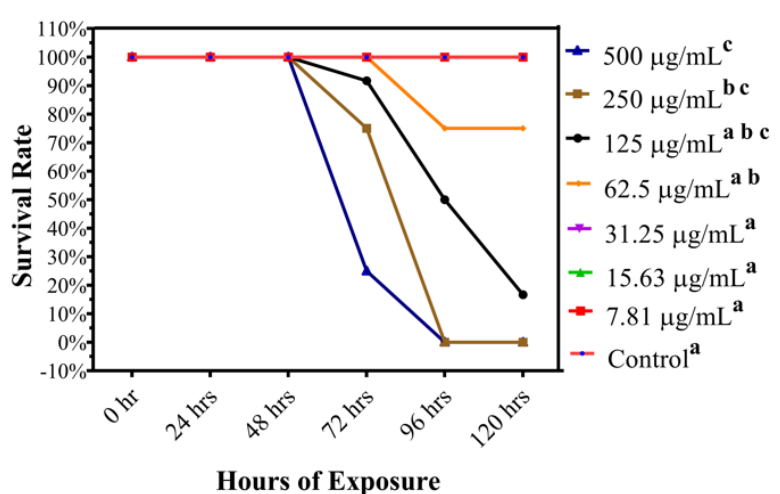

Figure 1. Effect of crude IL-GFE (7.81-500 $\mu \mathrm{g} / \mathrm{mL}$ ) on the survival rate of zebrafish embryos from 0 to $120 \mathrm{hpf}$. The sample was compared to the untreated group. The number of embryos tested for each concentration was 12 individually exposed embryos. The IL-GFE at a concentration of $31.25 \mu \mathrm{g} / \mathrm{mL}$ or less, showed no effect on the survival rate of zebrafish embryos. Superscripts with different letters (a-d) are significantly different $(\mathrm{p}<0.05)$.

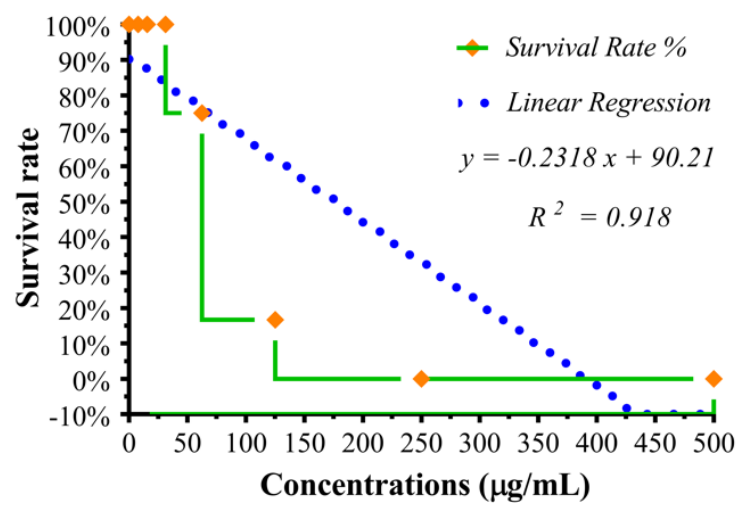

Figure 2. Effect of crude IL-GFE (7.81-500 $\mu \mathrm{g} / \mathrm{mL}$ ) on zebrafish embryos mortality rate at 120 hours post-fertilization (hpf). The $\mathrm{LC}_{50}$ value of crude IL-GFE towards zebrafish embryos was $173.45 \mu \mathrm{g} / \mathrm{mL}$. The number of embryos tested for each concentration was 12 individually exposed embryos.

\section{Effects of crude IL-GFE on morphology and development of zebrafish embryos}

Sublethal morphological and developmental effects were also assessed during the five days scoring period. The teratogenicity effects (Table 2) such as oedema (reversible side effect) and tail extension, somite formation, hatching rate and body curvature (non-reversible side effect) were observed for both control group and those treated with different concentrations of crude ILGFE at $72 \mathrm{hpf}$. It can be seen that the crude IL-
GFE at a concentration of less than $250 \mu \mathrm{g} / \mathrm{mL}$ did not show any effect on the tested teratogenicity parameters at $72 \mathrm{hpf}$. However, all larvae treated at a concentration of $500 \mu \mathrm{g} / \mathrm{mL}$ died with a sign of body curvature.

The microscopy observation showed that the lowest concentrations of the crude IL-GFE (less than $62 \mu \mathrm{g} / \mathrm{mL}$ ) did not affect the morphology (oedema, tail extension, somite formation, hatching rate and body curvature) of the treated larvae compared to the control group during the whole treatment period. In contrast, the crude IL-GFE at $500 \mu \mathrm{g} / \mathrm{mL}$ was found to be toxic to zebrafish embryos/larvae in which some embryos coagulated at $48 \mathrm{hpf}$, and all the larvae died at 96 hpf. Some larvae of these groups displayed an angle incidence of $0-45^{\circ}$ of the spinal cord curvature, which is considered as low body curvature.

Hatching process of zebrafish embryos was also used to assess the effect of the crude ILGFE on developing zebrafish embryos. Embryos hatching indicates the appropriate development of the embryos into larvae which usually occurs at 48 to $72 \mathrm{hpf}$ (Murugesu et al., 2019). The treated embryos did not show any significant differences in hatching rate when compared to the control group (Figure 4). The hatching rate of embryos treated with $500 \mu \mathrm{g} / \mathrm{mL}$ could not be measured due to the $75 \%$ mortality rate with coagulation at $72 \mathrm{hpf}$.

\section{Effects of crude IL-GFE on zebrafish heart rate}

Embryos and larvae were considered dead if no heartbeat was observed. Generally, the heart rate of zebrafish embryos/larvae ranges between 120 and $180 \mathrm{beat} / \mathrm{min}$ (Luca et al., 2014). The mean heart rates of zebrafish larvae were recorded at $96 \mathrm{hpf}$ and presented in Figure 5. The healthy larvae had a heart rate of $179.67 \pm 4.66 \mathrm{beat} / \mathrm{min}$ which is the highest rate, followed by the other treated larvae with the crude IL-GFE at concentrations of $7.81,15.63,31.25,62.5$ and 125 $\mu \mathrm{g} / \mathrm{mL}$, having heartbeat rates of $174.67,165.67$, 150.67, 134.00 and 119.00 beats $/ \mathrm{min}$, respectively while no heartbeat was recorded for the treated larvae with IL-GFE at the concentrations of 250 and $500 \mu \mathrm{g} / \mathrm{mL}$ due to early mortality. 
Table 2. Teratogenicity effects of crude IL-GFE $(7.81-500 \mu \mathrm{g} / \mathrm{mL})$ on zebrafish development at $72 \mathrm{hpf}$.

\begin{tabular}{cccccc}
\hline $\begin{array}{c}\text { Extract } \\
\begin{array}{c}\text { Concentrations } \\
(\mu \mathrm{g} / \mathrm{mL})\end{array}\end{array}$ & Oedema & Delayed hatch & $\begin{array}{c}\text { Not fully tail } \\
\text { extension }\end{array}$ & $\begin{array}{c}\text { Lack of somite } \\
\text { formation }\end{array}$ & $\begin{array}{c}\text { Body } \\
\text { curvature }\end{array}$ \\
\hline 7.81 & - & - & - & - & - \\
$\mathbf{1 5 . 6 3}$ & - & - & - & - & - \\
$\mathbf{3 1 . 2 5}$ & - & - & - & - & - \\
$\mathbf{6 2 . 5 0}$ & - & - & - & - & - \\
$\mathbf{1 2 5 . 0 0}$ & - & - & - & - & - \\
$\mathbf{2 5 0 . 0 0}$ & - & - & nd & - & - \\
$\mathbf{5 0 0 . 0 0}$ & nd & - & - & - & - \\
Control & - & - & &
\end{tabular}

(-): Not detected, (+): detected, (nd): Not determined.
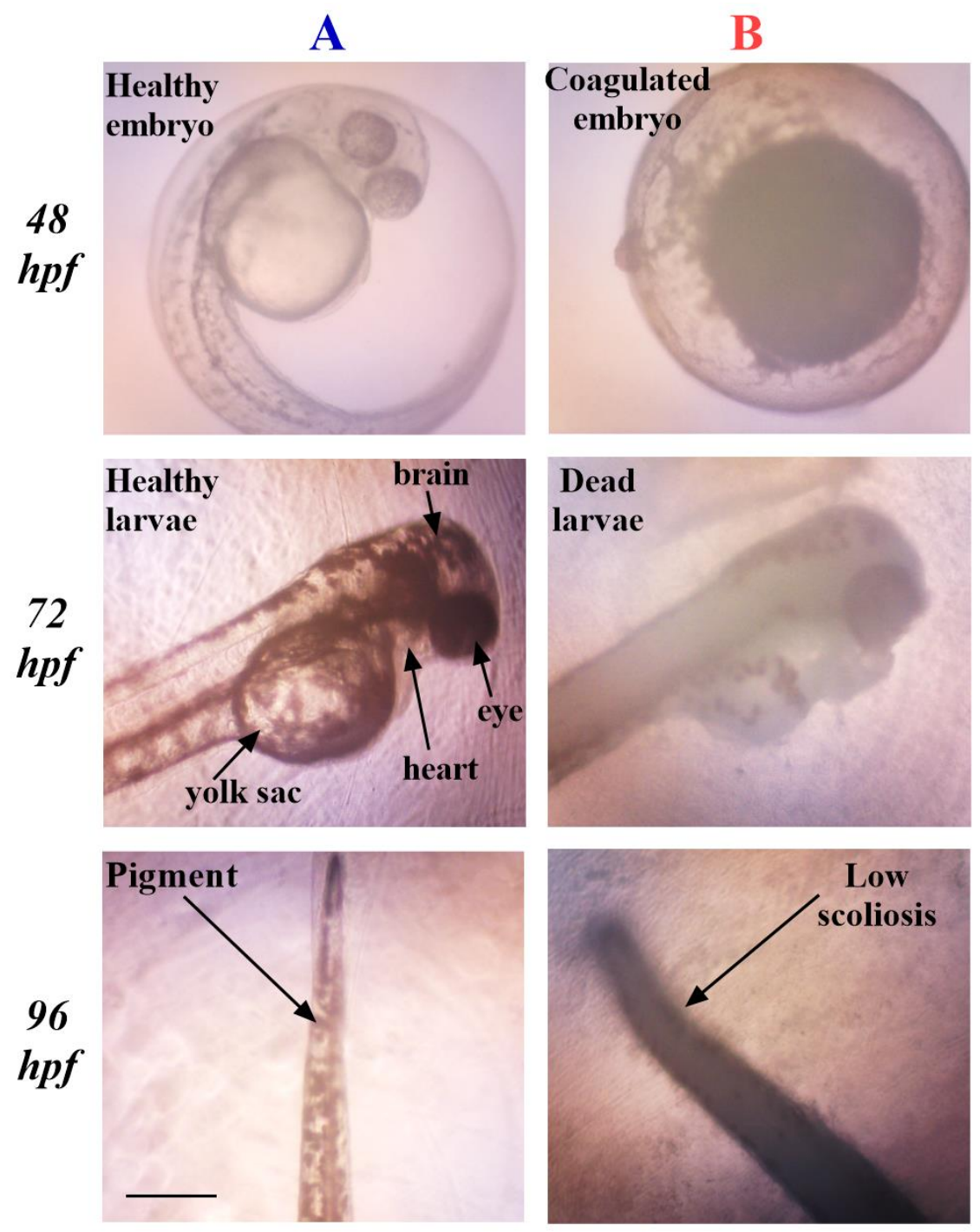

Figure 3. Representative photographs of A: untreated zebrafish (control) and B: the treated zebrafish with crude IL-GFE $(500 \mu \mathrm{g} / \mathrm{mL})$ at a designated time point. Photographs were captured using an inverted microscope at 100X magnification. Scale bar $=250 \mu \mathrm{m}$. 


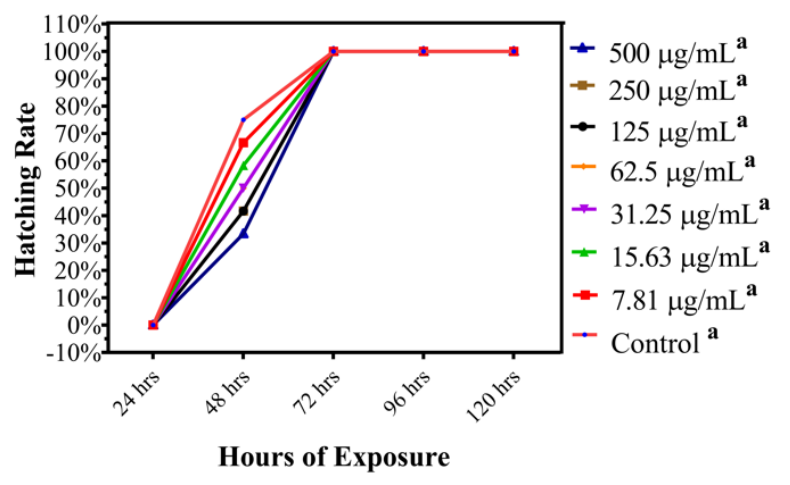

Figure 4. Effect of crude IL-GFE (7.81-500 $\mu \mathrm{g} / \mathrm{mL}$ ) on zebrafish embryos hatching rate at 0 to $120 \mathrm{hpf}$. The number of embryos tested for each concentration was 12 individually exposed embryos. Superscripts with different letters (a-d) are significantly different $(\mathrm{p}<0.05)$.

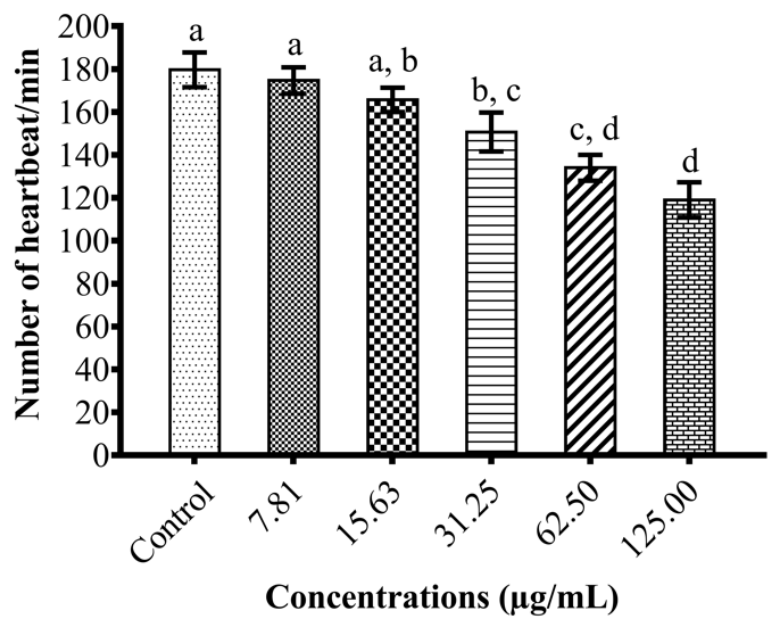

Figure 5. Heartbeat rates of zebrafish larvae treated with crude IL-GFE $(7.81-500 \mu \mathrm{g} / \mathrm{mL})$ at 96 hpf. Bars with different letters (a-d) are significantly different $(\mathrm{p}<0.05)$.

\section{DISCUSSION}

Secondary metabolites from plant sources have shown to be a promising potential therapeutic in various human diseases (Viraraghavan and Shanmugam, 2018). Phytochemical analysis of the crude IL-GFE revealed the presence of a complex mixture of secondary metabolites that are reported to have various medicinal activities (Gajalakshmi et al., 2012; Ojezele et al., 2016). These phytochemicals are acetogenins, alkaloids, flavonoids, tannins, terpenoids, and polyphenols. This finding is in line with the previous studies conducted on the ethanolic seed extract (Ojezele et al., 2016), ethanolic and water leaves extracts (Gavamukulya et al., 2014) and hydro-alcoholic fruit extract (Viraraghavan and Shanmugam, 2018) of Annona muricata that reported the existence of many secondary metabolites such as alkaloids, saponins, triterpenoids, anthraquinones, flavonoids, tannins, and cardiac glycosides. These metabolites are produced in the plant tissue and considered as defence compounds (Gavamukulya et al., 2014). Different identified metabolites from Graviola fruit such as acetogenins are believed to be mainly responsible for the anticancer activity of Graviola towards mammalian cancer cells (Mangal et al., 2016). However, some of the annonaceous acetogenins (AGE) was reported to have a significant induction of atypical parkinsonism in Guadeloupe Island (Höllerhage et al., 2015). These acetogenins are secondary metabolites deriving from the long-chained lipophilic in the polyketide pathway (Antony and Vijayan, 2016). They are inhibitors of the mitochondrial complex I by the inhibition of ubiquinone-linked $\mathrm{NADH}$ oxidase in the membrane (Escobar-Khondiker et al., 2007) causing apoptosis in cancer cells.

The toxicological effect of crude ionic liquidGraviola fruit extract was evaluated in vivo using developing zebrafish embryos as a model organism. The present study illustrates the usefulness of zebrafish as a useful model to rapidly assess the toxicity of crude IL-GFE. Many attributes make zebrafish as an attractive model for such studies. Zebrafish embryos grow up rapidly, and the major organs ultimately form within 48 hpf; hence, comprehensive toxicological assessment can be carried out within only a few days (Falcão et al., 2018). With the transparency nature of the embryos, many toxicological endpoints can be evaluated noninvasively during the experiment. Lethality rate, which is defined as the most sensitive endpoint, was assessed entirely within $48 \mathrm{~h}$ (Fraysse et al., 2006). Thus, influential dose-response studies are easily achieved through large sample sizes. This considerable supply of embryos also facilitates the assessment of toxicity of a significant number of materials in a short time.

Our present study demonstrated that crude IL-GFE had reduced the survival of zebrafish larvae at a relatively high dose of $250 \mu \mathrm{g} / \mathrm{mL}$ after $96 \mathrm{hpf}$ while no significant effect was observed at 
$62.5 \mu \mathrm{g} / \mathrm{mL}$ and lower concentrations. Previously, it was reported that any toxicants displaying an $\mathrm{LC}_{50}$ value range between 10 to $100 \mathrm{mg} / \mathrm{L}, 1$ to 10 $\mathrm{mg} / \mathrm{L}$ or less than $1 \mathrm{mg} / \mathrm{L}$ are considered highly toxic, toxic or harmful, respectively (Murugesu et al., 2019). Based on this scale, the crude IL-GFE is found to be non-harmful with an $\mathrm{LC}_{50}$ calculated to be $173.45 \mu \mathrm{g} / \mathrm{mL}$ on zebrafish embryo/larvae with bodyweight ranged between 58 and $79 \mu \mathrm{g}$ at 0 to $96 \mathrm{hpf}$ respectively (Hachicho et al., 2015).

Heart rates were recorded at $96 \mathrm{hpf}$ and showed a significant reduction of $33.76 \%$ at 125 $\mu \mathrm{g} / \mathrm{mL}$ as compared with the control group. A similar record was also registered in zebrafish larvae when treated with saxitoxin for $96 \mathrm{hrs}$ by Lefebvre et al. (2004). They suggested that oedema might be a consequence of a lack of movement and the observed decrease in heart rates may be a secondary effect of oedema and the alteration of fluid balance related to paralysis. Hatching is an essential process in the life cycle of fish, and many biochemical and physical mechanisms are involved in this process (Jin et al., 2009). In our study, the hatching rate of $48 \mathrm{hpf}$ average was in line with the standard hatchability of the zebrafish model, which is between 48 and $72 \mathrm{hpf}$ (Kimmel et al., 1995). Body curvature is another sign of malformation that was observed in zebrafish larvae after treatment with crude IL-GFE at a high concentration of $500 \mu \mathrm{g} / \mathrm{mL}$. Typically, there are three types of spinal curvature known as lordosis, kyphosis and scoliosis, that are classified based on the spinal bending degree. These spinal curvatures are caused by many factors, including toxins accumulation, inhibition of AChE enzyme activity and the deficit of neuromuscular coordination (Pamanji et al., 2015). Ekrem et al. (2012) and Hayes et al. (2014) suggested that spinal deformities may be caused by reduced amounts of collagen in the spinal column, downregulation of the protein tyrosine kinase seven (ptk7) that plays a vital role as a regulator of Wnt signalling and alteration of the amino acid composition.

Several plant metabolites were evaluated for their toxicity in zebrafish embryos/larvae such as curcumin (Wu et al., 2007), resveratrol (Pardal et al., 2014), caffeine (Chen et al., 2008), nicotine (Mora-Zamorano et al., 2016), cannabinoid (Akhtar et al., 2013), matrine and sophocarpine (Lu et al., 2014). Previously, Wu et al. (2007) have analysed the toxicity of curcumin (polyphenol derived from the rhizomes of Curcuma longa) on zebrafish embryos and larvae which displayed a significant decrease in survival and hatching rate at a dose of $7.5 \mu \mathrm{M}$ or higher. Another study was conducted to assess the toxicity of Millettia pachycarpa (Leguminosae) on zebrafish embryos in which the aqueous extract of this plant exhibited an $\mathrm{LC}_{50}$ of $3 \mu \mathrm{g} / \mathrm{mL}$. In addition to that, many developmental abnormalities, including pericardial oedema, muscle defects and body curvature, were observed in a dose-dependent manner (Yumnamcha et al., 2015).

Another study showed the toxicity of celastrol (terpenoid isolated from the Chinese herb Tripterygium wilfordii), which has been used traditionally as chemopreventive, neuroprotective and antioxidant agents (Kuchta et al., 2017). The toxicity of celastrol, which was evaluated on zebrafish embryos, showed that the concentration of $0.5 \mu \mathrm{M}$ or higher of celastrol induced many developmental abnormalities such as pericardial sac oedema, no blood flow and tail malformation. Also, the dose of $2.0 \mu \mathrm{M}$ of celastrol had killed all the treated embryos within $24 \mathrm{hrs}$ (Wang et al., 2011). Furthermore, the exposure of zebrafish embryos to matrine and sophocarpine (alkaloids obtained from Sophora alopecuroides) at a dose of 250 and $180 \mathrm{mg} / \mathrm{L}$, respectively exhibited frequent malformations at $96 \mathrm{hpf}$ that includes the appearance of tail bending, lack of hatching and loss of movement (Tropepe and Sive, 2003). However, matrine and sophocarpine are commonly used as anti-tumour, antiinflammatory, antioxidant and antiviral in Chinese traditional herbal medicine.

After analysing the data obtained from the acute toxicity studies conducted on mammals, Falcão et al. (2018) reported that the results of the toxicity test of cannabinoid exposure during pregnancy that was performed on zebrafish embryos (Akhtar et al., 2013) were comparable with the one observed in rodents (Taylor and Fennessy, 1977) and humans (El Marroun et al., 2010). They also found that cannabinoids can cause low birth weight, hyperactivity at small doses and low fetal growth in the treated animals. The use of these models in preclinical assays can be used to improve medicine through the identification of new pharmacological interventions at early stages of life. 


\section{CONCLUSION}

Phytochemical analysis for the crude ionic liquidGraviola fruit extract revealed the presence of many bioactive compounds such as acetogenins, alkaloids, phenols, flavonoids, tannins and terpenoids. These metabolites are known to have many therapeutic and physiological activities. The exposure to crude IL-GFE had reduced the survival of zebrafish larvae at a relatively high dose of $250 \mu \mathrm{g} / \mathrm{mL}$ after 96 hours postfertilization (hpf), which make it non-harmful to the zebrafish model. Interestingly, no significant changes on morphology of the treated zebrafish embryos/larvae with the crude IL-GFE at (125 $\mu \mathrm{g} / \mathrm{mL}$ ) have been recorded. However, heart rates were significantly reduced in the treated larvae with crude IL-GFE at $125 \mu \mathrm{g} / \mathrm{mL}$ as compared with the control group and a sign of low body curvature at a high dose treatment of $500 \mu \mathrm{g} / \mathrm{mL}$. Thus, further investigations are needed to understand and explain the specific effects of the Graviola fruit metabolites and build conclusions on human risk assessment.

\section{ACKNOWLEDGEMENTS}

Many thanks to the International Islamic University Malaysia (IIUM), for providing the PRIGS grant (P-RIGS18-065-0065) as well as to the Animal Biochemistry and Biotechnology Laboratory, Biochemistry Department, Faculy of Biotechnology and Biomolecular Sciences Universiti Putra Malaysia, for offering laboratory facilities to carry out this research.

\section{REFERENCES}

Abdul Ghafor, A. A. H., Elias, N., Shamsi, S., Yasin, F. M., \& Sarchio, S. N. E. 2020. Toxicity assessment of gallic acid loaded graphene oxide (GAGO) nano-formulation in zebrafish (Danio Rerio) embryos. Pertanika Journal of Science \& Tecbnology 28(1).

Akhtar, M. T., Ali, S., Rashidi, H., van der Kooy, F., Verpoorte, \& R., Richardson, M. K. 2013. Developmental effects of cannabinoids on zebrafish larvae. Zebrafish 10(3): 283-293.

Antony, P. \& Vijayan, R., 2016. Acetogenins from Annona muricata as potential inhibitors of antiapoptotic proteins: a molecular modeling study. Drug Design, Development and Therapy 10: 1399.
Ayoola, G., Coker, H., Adesegun, S., Adepoju-Bello, A., Obaweya, K., Ezennia, E. C., \& Atangbayila, T. 2008. Phytochemical screening and antioxidant activities of some selected medicinal plants used for malaria therapy in Southwestern Nigeria. Tropical Journal of Pharmaceutical Research 7(3): 10191024.

Bai, C. \& Tang, M. 2020. Toxicological study of metal and metal oxide nanoparticles in zebrafish. Journal of Applied Toxicology 40(1): 37-63.

Bhan, M., Satija, S., Garg, C., Dureja, H., \& Garg, M. 2017. A novel approach towards green extraction for glycyrrhitinic acid by ionic liquid based microwave assisted extraction and optimization through response surface methodology. Pharmacognosy Journal $9(6)$.

Bhat, S. V., Nagasampagi, B. A., \& Sivakumar, M. 2009. Natural products: chemistry and applications. Alpha Science International.

Chen, Y.-H., Huang, Y.-H., Wen, C.-C., Wang, Y.-H., Chen, W.L., Chen, L.-C., \& Tsay, H.-J. 2008. Movement disorder and neuromuscular change in zebrafish embryos after exposure to caffeine. Neurotoxicology and Teratology 30(5): 440-447.

Coria-Tellez, A. V., Montalvo-Gónzalez, E., Yahia, E. M., \& Obledo-Vázquez, E. N. 2018. Annona muricata: A comprehensive review on its traditional medicinal uses, phytochemicals, pharmacological activities, mechanisms of action and toxicity. Arabian Journal of Chemistry 11(5): 662691.

Daddiouaissa, D., Amid, A. 2018. Anticancer Activity of Acetogenins from Annona Muricata Fruit. International Medical Journal Malaysia 17(3): 103-112.

Daddiouaissa, D., Amid, A., Kabbashi, N. A., Fuad, F. A., Elnour, A. M., \& Epandy, M. A. 2019. Antiproliferative activity of ionic liquid-graviola fruit extract against human breast cancer (MCF-7) cell lines using flow cytometry techniques. Journal of Ethnopharmacology 236: 466-473.

Dai, Y., Hogan, S., Schmelz, E. M., Ju, Y. H., Canning, C., Zhou, K. 2011. Selective growth inhibition of human breast cancer cells by graviola fruit extract in vitro and in vivo involving downregulation of EGFR expression. Nutrition and Cancer 63(5): 795-801.

Economiques, O.d.c.e.d.d. 2013. Test No. 236: fish embryo acute toxicity (FET) test. OECD Publishing.

Ejikeme, C., Ezeonu, C. S., \& Eboatu, A. N. 2014. Determination of physical and phytochemical constituents of some tropical timbers indigenous to Nigerdelta area of Nigeria. European Scientific Journal 10(18): 247-270.

El Marroun, H., Tiemeier, H., Steegers, E. A., Roos-Hesselink, J. W., Jaddoe, V. W., Hofman, A., Verhulst, F. C., van den Brink, W., \& Huizink, A. C. 2010. A prospective study on intrauterine cannabis exposure and fetal blood flow. Early Human Development 86(4): 231-236.

Escobar-Khondiker, M., Höllerhage, M., Muriel, M.-P., Champy, P., Bach, A., Depienne, C., Respondek, G., Yamada, E. S., Lannuzel, A., \& Yagi, T. 2007. Annonacin, a natural mitochondrial complex I inhibitor, causes tau pathology in cultured neurons. Journal of Neuroscience 27(29): 7827-7837.

Evans, W. 1996. Trease and Evan's Pharmacognosy; 14th Edn London. England: WB Sounders company limited: 545-546.

Falcão, M. A. P., de Souza, L. S., Dolabella, S. S., Guimarães, A. G., \& Walker, C. I. B. 2018. Zebrafish as an alternative method for determining the embryo toxicity of plant products: a systematic review. Environmental Science and Pollution Research 25(35): 35015-35026.

Fraysse, B., Mons, R., \& Garric, J. 2006. Development of a zebrafish 4-day embryo-larval bioassay to assess toxicity of chemicals. Ecotoxicology and Environmental Safety 63(2): 253267. 
Gajalakshmi, S., Vijayalakshmi, S., \& Devi Rajeswari, V. 2012. Phytochemical and pharmacological properties of Annona muricata: a review. International Journal of Pharmacy and Pharmaceutical Sciences 4(2): 3-6.

Gavamukulya, Y., Abou-Elella, F., Wamunyokoli, F., \& El-Shemy, H. 2014. Phytochemical screening, anti-oxidant activity and in vitro anticancer potential of ethanolic and water leaves extracts of Annona muricata (Graviola). Asian Pacific Journal of Tropical Medicine 7: S355-S363.

Guadaño, A., Gutiérrez, C., de la Peña, E., Cortes, D., \& GonzálezColoma, A. 2000. Insecticidal and mutagenic evaluation of two annonaceous acetogenins. Journal of Natural Products 63(6): 773-776.

Gyamfi, K., Sarfo, D., Nyarko, B., Akaho, E., Serfor-Armah, \& Y., Ampomah-Amoako, E. 2011. Assessment of elemental content in the fruit of graviola plant, Annona muricata, from some selected communities in ghana by instrumental neutron activation analysis. Elixir Food Science 41: 56715675.

Hachicho, N., Reithel, S., Miltner, A., Heipieper, H. J., Küster, E., \& Luckenbach, T. 2015. Body mass parameters, lipid profiles and protein contents of zebrafish embryos and effects of 2, 4-dinitrophenol exposure. PloS One 10(8): $\mathrm{e} 0134755$.

Harborne, A. 1998. Phytochemical methods a guide to modern techniques of plant analysis. Springer Science \& Business Media.

Harborne, J. 1984. Phytochemical methods 2nd ed. Chapmann and Hall, London, New York: 156-157.

Hayes, M., Gao, X., Lisa, X. Y., Paria, N., Henkelman, R. M., Wise, C. A., \& Ciruna, B. 2014. ptk7 mutant zebrafish models of congenital and idiopathic scoliosis implicate dysregulated Wnt signalling in disease. Nature Communications 5: 4777.

Hill, A. J., Teraoka, H., Heideman, W., \& Peterson, R. E. 2005. Zebrafish as a model vertebrate for investigating chemical toxicity. Toxicological sciences 86(1): 6-19.

Höllerhage, M., Rösler, T. W., Berjas, M., Luo, R., Tran, K., Richards, K. M., Sabaa-Srur, A. U., Maia, J. G. S., Moraes, M. R. D., \& Godoy, H. T. 2015. Neurotoxicity of dietary supplements from Annonaceae species. International Journal of Toxicology 34(6): 543-550.

Jin, M., Zhang, X., Wang, L., Huang, C., Zhang, Y., \& Zhao, M. 2009. Developmental toxicity of bifenthrin in embryo-larval stages of zebrafish. Aquatic Toxicology 95(4): 347-354.

Kapp, T., Kammann, U., Vobach, M., \& Vetter, W. 2006. Synthesis of low and high chlorinated toxaphene and comparison of their toxicity by zebrafish (Danio rerio) embryo test. Environmental Toxicology and Chemistry: An International Journal 25(11): 2884-2889.

Kimmel, C. B., Ballard, W. W., Kimmel, S. R., Ullmann, B., \& Schilling, T. F. 1995. Stages of embryonic development of the zebrafish. Developmental Dynamics 203(3): 253-310.

Kjærstad, G., Webb, J. M., \& Ekrem, T. 2012. A review of the Ephemeroptera of Finnmark-DNA barcodes identify Holarctic relations. Norwegian Journal of Entomology 59(2): 182-195.

Kuchta, K., Xiang, Y., Huang, S., Tang, Y., Peng, X., Wang, X., Zhu, Y., Li, J., Xu, J., \& Lin, Z. 2017. Celastrol, an active constituent of the TCM plant Tripterygium wilfordii Hook. f., inhibits prostate cancer bone metastasis. Prostate Cancer and Prostatic Diseases 20(2): 156.

Lannuzel, A., Michel, P., Höglinger, G., Champy, P., Jousset, A., Medja, F., Lombes, A., Darios, F., Gleye, C., \& Laurens, A. 2003. The mitochondrial complex I inhibitor annonacin is toxic to mesencephalic dopaminergic neurons by impairment of energy metabolism. Neuroscience 121(2): 287296
Lefebvre, K. A., Trainer, V. L., \& Scholz, N. L. 2004. Morphological abnormalities and sensorimotor deficits in larval fish exposed to dissolved saxitoxin. Aquatic Toxicology 66(2): 159-170.

Lieschke, G. J. \& Currie, P. D. 2007. Animal models of human disease: zebrafish swim into view. Nature Reviews Genetics 8(5): 353.

Lu, Z.-G., Li, M.-H., Wang, J.-S., Wei, D.-D., Liu, Q.-W., \& Kong, L.-Y. 2014. Developmental toxicity and neurotoxicity of two matrine-type alkaloids, matrine and sophocarpine, in zebrafish (Danio rerio) embryos/larvae. Reproductive Toxicology 47: 33-41.

Luca, E. D., Zaccaria, G. M., Hadhoud, M., Rizzo, G., Ponzini, R., Morbiducci, U., \& Santoro, M. M. 2014. ZebraBeat: a flexible platform for the analysis of the cardiac rate in zebrafish embryos. Scientific Reports 4: 4898.

Mangal, M., Imran Khan, M., \& Mohan Agarwal, S. 2016. Acetogenins as potential anticancer agents. Anti-Cancer Agents in Medicinal Chemistry (Formerly Current Medicinal Chemistry-Anti-Cancer Agents) 16(2): 138-159.

Mora-Zamorano, F. X., Svoboda, K. R., \& Carvan III, M. J. 2016. The nicotine-evoked locomotor response: a behavioral paradigm for toxicity screening in zebrafish (Danio rerio) embryos and eleutheroembryos exposed to methylmercury. PloS One 11(4).

Murugesu, S., Khatib, A., Ahmed, Q. U., Ibrahim, Z., Uzir, B. F., Benchoula, K., Yusoff, N. I. N., Perumal, V., Alajmi, M. F., \& Salamah, S. 2019. Toxicity study on Clinacanthus nutans leaf hexane fraction using Danio rerio embryos. Toxicology Reports 6: 1148-1154.

Ng'uni, T., Klaasen, J. A., \& Fielding, B. C. 2018. Acute toxicity studies of the South African medicinal plant Galenia africana. Toxicology Reports 5: 813-818.

Ojezele, O. J., Ojezele, M. O., \& Adeosun, A. M. 2016. Comparative phytochemistry and antioxidant activities of water and ethanol extract of Annona muricata Linn Leaf, seed and fruit. Advances in Biological Research 10(4): 230-235.

Oliveira, R. C. S., Oliveira, R., Rodrigues, M. A. C., de Farias, N. O., Sousa-Moura, D., Nunes, N. A., Andrade, T. S., \& Grisolia, C. K. 2020. Lethal and Sub-lethal Effects of Nitrofurantoin on Zebrafish Early-Life Stages. Water, Air, \& Soil Pollution 231(2): 54.

Oliver, A. L.-S., Sanz-Landaluze, J., Muñoz-Olivas, R., Guinea, J., \& Cámara, C. 2011. Zebrafish larvae as a model for the evaluation of inorganic arsenic and tributyltin bioconcentration. Water Research 45(19): 6515-6524.

Ong, E. S., Chor, C. F., Zou, L., \& Ong, C. N. 2009. A multianalytical approach for metabolomic profiling of zebrafish (Danio rerio) livers. Molecular BioSystems 5(3): 288-298.

Pamanji, R., Yashwanth, B., Bethu, M., Leelavathi, S., Ravinder, K., \& Rao, J.V. 2015. Toxicity effects of profenofos on embryonic and larval development of Zebrafish (Danio rerio). Environmental Toxicology and Pharmacology 39(2): 887897.

Pardal, D., Caro, M., Tueros, I., Barranco, A., \& Navarro, V. 2014. Resveratrol and piceid metabolites and their fat-reduction effects in zebrafish larvae. Zebrafish 11(1): 32-40.

Parichy, D. M., Elizondo, M. R., Mills, M. G., Gordon, T. N., \& Engeszer, R. E. 2009. Normal table of postembryonic zebrafish development: staging by externally visible anatomy of the living fish. Developmental Dynamics 238(12): 2975-3015.

Ragasa, C. Y., Soriano, G., Torres, O. B., Don, M.-J., \& Shen, C.C. 2012. Acetogenins from Annona muricata. Pharmacognosy Journal 4(32): 32-37.

Romagosa, C. M. R., David, E. S., \& Dulay, R. M. R. 2016. Embryo-toxic and teratogenic effects of Tinospora cordifolia 
leaves and bark extracts in Zebrafish (Danio rerio) embryos. Asian Journal on Plant Sciences and Research 6(2): 37-41.

Scarlett, A. G., Reinardy, H. C., Henry, T., West, C. E., Frank, R. A., Hewitt, L. M., \& Rowland, S. J. 2013. Acute toxicity of aromatic and non-aromatic fractions of naphthenic acids extracted from oil sands process-affected water to larval zebrafish. Chemosphere 93(2): 415-420.

Sousa, D. O. V., Vieira, G. D.-V., De Pinho, J. D. J. R., Yamamoto, C. H., \& Alves, M. S. 2010. Antinociceptive and antiinflammatory activities of the ethanol extract of Annona muricata L. leaves in animal models. International Journal of Molecular Sciences 11(5): 2067-2078.

Sun, S., Liu, J., Sun, X., Zhu, W., Yang, F., Felczak, L., Dou, Q. P., \& Zhou, K. 2017. Novel Annonaceous acetogenins from Graviola (Annona muricata) fruits with strong antiproliferative activity. Tetrahedron Letters 58(19): 1895-1899.

Tang, B., Bi, W., Tian, M., \& Row, K. H. 2012. Application of ionic liquid for extraction and separation of bioactive compounds from plants. Journal of Chromatography B 904: 1-21.

Taylor, D. A. \& Fennessy, M. R. 1977. Biphasic nature of the effects of $\Delta$-tetrahydrocannabinol on body temperature and brain amines of the rat. European Journal of Pharmacology 46(2): 93-99.

Tropepe, V. \& Sive, H. L. 2003. Can zebrafish be used as a model to study the neurodevelopmental causes of autism? Genes, Brain and Behavior 2(5): 268-281.

Viraraghavan, S. \& Shanmugam, N. 2018. Phytochemical screening of hydroalcohol fruit extract of Annona muricata. International Journal of Advanced Research and Development 3(1): 681-685.

Vyas, B. A. 2010. Phytopharmacological action of pergularia daemia with special reference to its actions and mechanism of action as diuretic and anti inflammatory agent $\mathrm{PhD}$ thesis). Veer Narmad South Gujarat University.

Wang, S., Liu, K., Wang, X., He, Q., \& Chen, X. 2011. Toxic effects of celastrol on embryonic development of zebrafish (Danio rerio). Drug and Chemical Toxicology 34(1): 61-65.

Wu, J.-Y., Lin, C.-Y., Lin, T.-W., Ken, C.-F., \& Wen, Y.-D. 2007. Curcumin affects development of zebrafish embryo. Biological and Pharmacentical Bulletin 30(7): 1336-1339.

Yan, L., Gong, C., Zhang, X., Zhang, Q., Zhao, M., \& Wang, C. 2016. Perturbation of metabonome of embryo/larvae zebrafish after exposure to fipronil. Environmental Toxicology and Pharmacology 48: 39-45.

Yumnamcha, T., Roy, D., Devi, M. D., \& Nongthomba, U. 2015. Evaluation of developmental toxicity and apoptotic induction of the aqueous extract of Millettia pachycarpa using zebrafish as model organism. Toxicological \& Environmental Chemistry 97(10): 1363-1381.

Yunus, A. B. M., Wan Chik, W., \& Mohamad, M. B. 2010. The concept of halalan tayyiba and its application in products marketing: A case study at Sabasun HyperRuncit Kuala Terengganu, Malaysia. International Journal of Business and Social Science 1(3): 239-248.

Zakaria, K. N., Amid, A., Zakaria, Z., Jamal, P., \& Ismail, A. 2019. Anti-proliferative activity of triterpenes isolated from Clinicanthus nutans on Hep-G2 liver cancer cells. Asian Pacific Journal of Cancer Prevention: APJCP 20(2): 563.

Zhang, Y., Liu, Z., Li, Y., \& Chi, R. 2014. Optimization of ionic liquid-based microwave-assisted extraction of isoflavones from Radix puerariae by response surface methodology. Separation and Purification Technology 129: 71-79.

Zohra, S. F., Meriem, B., Samira, S., \& Muneer, M. A. 2012. Phytochemical screening and identification of some compounds from mallow. Journal of Natural Product and Plant Resources 2(4): 512-516. 